\title{
SCATTERED LIGHT IN A DISTANT RADIO GALAXY
}

\author{
ANDREA CIMATTI \\ ESO (Germany) and Dipartimento di Astronomia, Firenze (Italy) \\ and \\ SPERELLO DI SEREGO ALIGHIERI \\ Osservatorio Astrofisico di Arcetri, Firenze (Italy)
}

\begin{abstract}
We present optical polarimetric observations of a radio galaxy at $\mathrm{z}=2.63$ and the results of its spectral modelling made by using stellar and scattered anisotropic nuclear radiation.
\end{abstract}

\section{Polarimetric observations}

High redshift radio galaxies (HZRG) [2] provide a unique possibility to study galaxies at early cosmological epochs. An accurate separation of the stellar and nonstellar components is necessary to infer the evolutionary status of these galaxies. In order to derive the ratio between the stellar and the non-stellar radiation we have observed a very distant radio galaxy by CCD imaging polarimetry.

Polarimetric observations of the radio galaxy MRC2025-218 were made at 9 different position angles in $\mathrm{R}$ band $\left(\lambda_{\text {rest }} \sim 1900 \AA\right)$ at the ESO/MPG $2.2 \mathrm{~m}$ and ESO $3.6 \mathrm{~m}$ telescopes. We detect high linear polarization $\left(P=8.3 \pm 2.3 \%, \theta=93^{\circ} \pm 8^{\circ}\right)$ with the $\mathbf{E}$-vector perpendicular to the radio-optical axis. The polarization properties are consistent with the ones of radio galaxies at $0.5<\mathrm{z}<1.2$ [1]. The presence of high polarization indicates that the UV radiation is not purely stellar and that a realistic modelling of the spectral energy distribution (SED) must include both stellar and non-stellar radiation.

\section{Spectral and polarization modelling}

The high perpendicular polarization suggests that the UV radiation is due to scattering of anisotropic nuclear radiation rather than to a jet-induced starburst phenomenon [1]. We have successfully fitted both the degree of polarization and the SED between 1000-6000 $\AA$ by using two components : an evolved host galaxy (age $\sim 2 \mathrm{Gyr}$ ) and a dust (Galactic grains) scattered quasar spectrum (scattering angle $45^{\circ}$ ). According to this model, all the UV radiation is due to scattering of the light coming from the obscured quasar nucleus. Our result is consistent with the radio loud quasars - radio galaxies unifying scheme.

\section{References}

1. Cimatti A., di Serego Alighieri S., Fosbury R., Salvati M., Taylor D., 1993, MNRAS, 264, 421.

2. McCarthy P.J. 1993 ARAA, in press

T. J.-L. Courvoisier and A. Blecha: Multi-Wavelength Continuum Emission of AGN, 468.

(C) 1994 IAU. Printed in the Netherlands. 